\title{
La Citología de Cuello Uterino en Soacha, Colombia: Representaciones Sociales, Barreras y Motivaciones
}

Carolina Wiesner-Ceballos ${ }^{1}$, Marcela Vejarano-Velandia² ${ }^{2}$ Juan C. Caicedo-Mera ${ }^{3}$, Sandra L. Tovar-Murillo ${ }^{4}$ y Ricardo Cendales-Duarte ${ }^{5}$

${ }^{1}$ Médica. M. Sc. Salud Pública. Instituto Nacional de Cancerología. Universidad Externado de Colombia.E-mail: cwiesner@cancer.gov.co

${ }^{2}$ Trabajadora Social. M. Sc. Derechos de Infancia y Adolescencia. Centro de Investigaciones Sociales (CIDS), Universidad Externado de Colombia. E-mail: marcela.vejarano@uexternado.edu.co

${ }^{3}$ Médico. Centro de Investigaciones Sociales (CIDS), Universidad Externado de Colombia. E-mail:

juan.caicedo@uexternado.edu.co
${ }^{4}$ Enfermera. Especialista en Administración de Servicios de Salud. Instituto Nacional de Cancerología, Bogotá, Colombia. E-mail: stovar@cancer.gov.co

${ }^{5}$ Médico. M. Sc. Epidemiología. Instituto Nacional de Cancerología, Bogotá, Colombia. E-mail: rcendales@cancer.gov.co Recibido 5 Abril 2006/Enviado para Modificación 6 Junio 2006/Aceptado 21 Octubre 2006

\section{RESUMEN}

Objetivos Presentar las representaciones sociales de la citología así como las barreras y las motivaciones, como punto de partida, para adecuar los servicios de prevención en un municipio de Colombia.

Métodos Estudio cualitativo de tipo fenomenológico mediante el uso de grupos focales y talleres de validación. Se realizó un muestreo teórico para buscar diferencias según la edad, el tipo de afiliación al sistema de salud, antecedente de citología y tipo de comuna. Se realizó interpretación de las narrativas de las participantes y análisis mediante el uso de software Atas ti. Resultados La citología se asocia con la idea de infecciones, pre-cáncer y prevención de cáncer. Se identificaron barreras culturales, desinformación por pares, ausencia de redes sociales y, particularmente, mala calidad de algunos servicios de salud del sector público.

Discusión El proceso de objetivación frente al examen citológico, se produce de manera satisfactoria aunque no hay una relación univoca entre conocimiento y práctica continua de la citología. Las mujeres mayores tienen representaciones de riesgo propio del modelo de las infecciones agudas La falta de oportunidad en la asignación de citas y entrega de resultados afecta la continuidad necesaria para el control del cáncer.

Palabras Clave: Representaciones sociales, citología, cuello uterino, garantía de la calidad de la atención (fuente: MeSH, BIREME).

\section{ABSTRACT \\ Cervical cytology in Soacha, Colombia: social representation, barriers and motivation}


Objective A social representation of the Pap smear is given in this article, as are the barriers against it and the motivation for practising it, aimed at adjusting preventative health services in a particular Colombian town.

Methods A phenomenological, qualitative study was carried out, using focal groups and workshops for validating the results. A theoretical sample was designed to find differences according to age, heatlh system affiliation and dwelling place within the municipality in question. Atlas ti software was used for giving the analysis a narrative interpretation.

Results The Pap smear is associated with infection and pre-cancer and cancer prevention. Cultural barriers, misinformation being spread by peers, a lack of social networks and the particularly poor quality of Colombian public health services were identified.

Discussion Attempts to make the Pap smear as objective as possible were found to be satisfactory, even though no logical relationship was found between knowledge of the Pap smear and its practice. Older women have the social representation of risk implicit in an acute infection model. Lack of opportunity for a having a Pap smear appointment and delays in presenting the corresponding report have a negative impact on cancer control.

Key Words: Social representation, cytology, cervix uteri, health care quality assurance (source: $\mathrm{MeSH}, \mathrm{NLM}$ ).

$\mathbf{E}$ n el mundo, el carcinoma de cuello uterino es el segundo tipo de cáncer que afecta con mayor frecuencia a la mujer, especialmente en los países con bajo desarrollo económico (1). En América Latina, la mortalidad femenina se ha incrementado por esta causa, con excepción de México y Chile, que presentan el mayor ingreso per cápita de la región y el mejor rendimiento de los indicadores económicos en general (2). Los programas para la prevención y el diagnóstico precoz de cáncer de cuello uterino no han tenido un adecuado impacto en la reducción mortalidad en la mayoría de los países con bajo desarrollo económico $(3,4,5,6)$.

En Colombia, la citología cérvico-uterina se ha promovido desde hace más de 30 años y la cobertura se ha considerado aceptable según la prevalencia encontrada en 1998 (68,4 \%) (7) y en 2005 (75 \%) (8). Sin embargo, el cáncer de cuello uterino continúa siendo un problema importante de salud pública pues representa la segunda causa de muerte por cáncer en mujeres en Colombia (Tasa de mortalidad ajustada por subregistro de 18,1 por 100000 mujeres) (1). Probablemente factores relacionados con barreras de acceso, con una falta de adecuación de los servicios de salud a la forma como se distribuye el riesgo en la población de mujeres, además con la estructura y organización del actual Sistema 
General de Seguridad Social en Salud (SGSSS) parecen explicar este hecho (9, $10,11)$.

En este contexto se consideró necesario conocer la realidad que viven las mujeres con mayor riesgo para cáncer de cuello uterino; se seleccionó el municipio de Soacha en el departamento de Cundinamarca porque agrupa población de bajo nivel socioeconómico, con heterogeneidad cultural y con diferentes niveles de acceso a los servicios de salud; también porque está ubicado a pocos kilómetros de Bogotá.

Teniendo en cuenta que las representaciones sociales, como fenómenos cognitivos, "relacionan la pertenencia social de los individuos, con la interiorización de experiencias, prácticas y modelos de comportamiento y pensamiento que le están asociadas" (12) se consideró necesario, mediante esta perspectiva, conocer el significado que tiene la citología y el cáncer de cuello uterino, así como las barreras y las motivaciones para utilizar los servicios de prevención. Dos procesos han sido descritos dentro del soporte teórico de la dinámica de construcción de las representaciones sociales $(13,14)$; uno de objetivación, en el cual los contenidos se hacen transparentes al ser reformulados en el lenguaje de la comunidad, y otro, de anclaje, en el cual estos contenidos se incorporan al pensamiento colectivo, impregnados de formas valorativas que pueden orientar conductas sociales específicas.

\section{MATERIALES Y MÉTODOS}

Este estudio hace parte de un proyecto más amplio titulado "Evaluación de una estrategia para inducción de la demanda de servicios de tamizaje de cáncer de cuello uterino en dos comunas del municipio de Soacha". Para esta fase se seleccionó una metodología cualitativa de tipo fenomenológico mediante el uso de grupos focales y talleres de validación de resultados. Para la selección del número de mujeres se utilizó la técnica del muestreo teórico $(15,16)$. La pregunta que originó el muestreo fue la siguiente: ¿Existen diferencias en las percepciones de la citología, y de las barreras y motivaciones para su toma, de acuerdo con la edad de la mujer, el tipo de afiliación al SGSSS, el lugar de residencia y el antecedente de citología en los últimos tres años? La edad, por cuanto las mujeres menores de 35 años tienen mayor probabilidad de contacto con los servicios de salud por razones de su reproducción (17); el tipo de afiliación, puesto que se ha encontrado en Colombia que la mortalidad de cáncer de cuello uterino es más alta en las mujeres que no tienen ningún tipo de afiliación al sistema de seguridad 
social en salud. El antecedente de citología, pues las mujeres que se hacen la citología han tenido alguna motivación para hacérsela. El lugar de residencia, por cuanto las comunas del Municipio seleccionado presentan claras diferencias socioeconómicas.

Las mujeres afiliadas al régimen subsidiado, se localizaron a través de líderes comunitarias con la técnica de bola de nieve. Para localizar mujeres sin ningún tipo de afiliación, se acudió a la Secretaría de Salud del Municipio, quien suministró el censo de las mujeres. De los listados iniciales se hizo una selección final de acuerdo con la edad y con la información suministrada respecto a la realización de la citología en los últimos tres años. Finalmente, se realizó llamada telefónica inicial y posteriormente invitación personalizada.

Se realizaron 6 grupos focales con un promedio de asistencia de 8 mujeres por grupo. El porcentaje de participación fue del $95 \%$. De las 48 mujeres el 30 \% eran menores de 35 años y el 70 \% mayores de 35 años La mitad estaba afiliada al régimen subsidiado y la otra mitad no tenía ningún tipo de afiliación. El 55\% no tenía antecedente de citología en los últimos tres años; la mitad de las mujeres eran residentes de la comuna 2; la otra mitad residentes de la comuna 4. Entre diciembre de 2005 y febrero de 2006 se hicieron seis talleres de validación de la información con 39 mujeres que cumplían las mismas características anteriores. En estos, se presentaron los principales hallazgos encontrados, y las mujeres debían opinar y profundizar en el tema de acuerdo con las categorías.

Para la recolección de la información, de los grupos focales y los talleres de validación, se llevó un registro sonoro en medio magnético, el cual se transcribió para su análisis. Cada sesión tuvo una duración aproximada de dos horas. Los grupos fueron dirigidos por profesionales de las ciencias sociales; la relatoría y la observación la realizaron profesionales de la salud y de las ciencias sociales. Por ser un estudio de riesgo mínimo y de acuerdo con la Resolución 8430 de 1993 se solicitó consentimiento verbal para la grabación y se garantizó la confidencialidad de la información.

Se hizo un análisis preliminar de manera simultánea a la recolección. Para cada pregunta se buscó alcanzar la saturación teórica. Los textos fueron procesados por medio del software Atlas-ti; con éste, se crearon filtros de análisis por grupo de edad, tipo de afiliación, antecedente de citología, tipo de comuna y se buscaron diferencias de contenido entre estas categorías. 


\section{RESULTADOS}

Representación social de la citología de cuello uterino

Las mujeres del estudio expresaron que "la citología sirve para saber cómo estamos por dentro" porque "uno se cuida por fuera, pero no sabe como está por dentro". Estas expresiones manifiestan la inquietud por acceder a un espacio del cuerpo que es invisible y sólo deja de ser ajeno por acción de la práctica médica. El paso del conocimiento científico al dominio público se produce en términos del develamiento de una corporalidad íntima y oculta a través de los ojos de otro que es el profesional de la salud: "Yo entiendo que es como prevención, como investigación muy profunda, a nivel de que uno no puede verse por allá ni nada, pues es otro método que está analizando más profundo para ver qué encuentran o cómo va uno, es parte de quererse a sí mismo". De acuerdo con las narrativas, la citología es una prueba que permite anticiparse a la enfermedad o descubrirla en su estado oculto.

En este espacio invisible como es la matriz, la citología sirve para detectar todo lo que sucede en ella: las infecciones, cáncer y "pre-cancer": "La citología sirve para ver si hay flujo, creo yo." "Puede uno tener una simple infección, a mí me dijeron tiene una infección y al rato me dijeron: no, tiene pre-cáncer". "Sí, la citología se asocia con cáncer uterino". Las infecciones de la matriz se asocian también con el uso del dispositivo intrauterino: "El dispositivo le da a uno una infección, entonces puede ser por eso, o de pronto también venéreas, no se".

Se encontró que no todas las mujeres mayores de treinta y cinco años, se realizan la citología a pesar de conocer su utilidad: "Pues yo creo que no la necesitaba no, porque yo no tenía relaciones, me mantenía sola, pendiente de mis hijos, y pues nunca me enfermaba de nada".

El núcleo figurativo de la representación de la 'citología' centrado en la matriz y en el cáncer, proviene del discurso científico difundido desde hace varias décadas en las campañas de prevención y de una red de comunicaciones provenientes de familiares, vecinas o allegadas, que convierten el examen en una temática presente en la comunidad. Esta simbiosis de fuentes de referencia configura un complejo campo de intersección entre contenidos puramente racionales, presupuestos valorativos y trasfondos emocionales, dado que se trata de una problemática asociada con la muerte y con diversos aspectos de la intimidad sexual. No se encontraron diferencias entre los grupos de mujeres descritos en el muestreo.

Barreras para la toma de la citología

Las barreras culturales para la toma de la citología se hacen evidentes cuando las mujeres actúan priorizando lo urgente (el cuidado de los demás, de la casa, y del 
trabajo) en lugar de lo importante, como podría ser el autocuidado de la salud: "no me ha quedado tiempo porque he estado con la niña..." El tabú hacia la sexualidad, refuerza los sentimientos de vergüenza y el temor que genera la desnudez y la exposición de las partes íntimas: "Pues a mi me da pena, a mi siempre me ha dado pena, porque uno tiene que dejarse, uno bajarse la ropa interior, tiene que mirarme, uno sus partes íntimas, entonces, a mi me pasa eso..." No se encontraron diferencias entre las mujeres por edad, tipo de afiliación al sistema de salud, lugar de residencia, ni antecedente de citología.

La información que reciben las mujeres del vecindario acerca de la citología como un examen doloroso o traumático es un obstáculo para su realización: "Pues uno habla con las personas, siempre uno tiende a recibir todo lo malo que lo bueno (...), no, eso duele, que eso le cortan, le sacan un pedazo de la matriz, entonces uno lleva ya una imagen en la mente y cuando llega allá, me va a doler, me va a doler y, pues claro."; "salían las otras señoras que habían entrado primero, que contaban, que eso duele, que yo salí sangrando". Los contenidos provenientes de la información médica, no siempre llevan a la práctica de la citología porel temor generado ante el conocimiento de una experiencia desagradable relatada por un par.

La pareja se puede convertir en un obstáculo para la toma de la citología. Según las mujeres, algunos hombres desconfían de su pareja, al considerar que en lugar de ir al médico la mujer se dirige a otro lugar, o cuando cree que la toma de citología implica el "manoseo" a la mujer por parte del médico.: "Eso era pelea, cuando yo iba donde el médico él decía que yo no iba para donde el médico, sino para donde el mozo $(\ldots)$

La ausencia de redes sociales limita la posibilidad de acudir al médico; esta ausencia de redes es mayor en aquellas mujeres que han sido víctimas de desplazamiento forzado por la violencia. "(...) y más si uno no tiene conocidos desplazados, en la casa soy desplazada. Uno dice: ¡ni con la vecina dejo las niñas! ¡Usted viera para salir...!"

Las familias de las jóvenes o de las mujeres solteras se constituyen en una barrera para la toma por cuanto implica reconocer el inicio de la vida sexual frente a los padres; una situación similar ocurre con maestros y compañeros de colegio.

Calidad de los servicios. Las mujeres describen múltiples obstáculos de acceso. Ellas expresaron la necesidad de realizar largas filas o trámites dispendiosos para acceder al servicio. Estos problemas se extienden también al proceso de entrega de resultados.:"la entrega de resultados no me gustó, porque como tres meses duré yendo cada mes a reclamar y no, que todavía no estaban, que todavía no. Ahí entonces vine como hasta los cinco meses y las reclame, y hasta ahorita vengo a traerla porque no había pasado antes a que me la vieran."; "Y también las madrugadas porque tiene uno que ir a perder el tiempo por decir, uno pierde un trabajo y entonces no le dan permiso en primer lugar, y entonces uno dice no, pues, está primero 
el trabajo, me echan y entonces qué (...)". Dadas las barreras para el acceso, algunas mujeres prefieren recurrir a servicios privados o simplemente no tomarse la citología.

En los centros de atención para las no aseguradas, la relación médico paciente es pobre, no solo por la corta duración de las consultas, sino además, por el trato por parte de funcionarios, que es percibido como duro e inhumano. A los sentimientos de temor y vergüenza ante la toma de citología, se suma el hecho de que los funcionarios dan mínimas explicaciones sobre el procedimiento. Hay una desarticulación en la información que se brinda sobre las rutas de atención.

Algunas mujeres sin antecedente reciente de citología, decidieron no volver a hacérsela para no exponerse a la tramitología, a la pérdida de tiempo y dinero, al maltrato, a la demora en la entrega o pérdida del resultado del examen, entre otros motivos. Por otra parte las mujeres jóvenes sin afiliación ni antecedente de citología manifestaron que el no tener la carta del SISBEN es determinante para su no realización. Las mujeres mayores de 35 años, no afiliadas acuden a la consulta particular antes que a los servicios públicos.

Motivaciones para la toma de citología

El autocuidado y la preocupación por la familia. Las mujeres menores se realizan la citología por considerar que es una responsabilidad hacérsela.: "sé que tengo que hacerlo y lo he hecho porque es un bien para mi salud; además, porque yo todavía no he tenido hijos y quiero estar bien para cuando vaya a tener un hijo". Las mujeres mayores manifestaron las expectativas que tienen frente a su vida en el futuro: "Para llegar a una vejez,(...) hay que ser constante con el control, así a uno no le duela ni un solo dedo".

Algunas mujeres mostraron desconfianza o suspicacia frente a sus compañeros sexuales y esto se convirtió en una motivación hacia la toma de la citología; “(...) por ejemplo la citología es muy importante, para uno de mujer, para estar pendiente, qué enfermedades le puede transmitir el esposo a uno"; en otras palabras, la eventualidad del contagio, en mujeres con vida sexual activa y el subsecuente sentido de responsabilidad motiva la conducta preventiva. La valoración causal de la relación contagio-sexualidad no sólo parece estar asociada con el comportamiento de la propia mujer, sino que se extiende a las conductas de los cónyuges o compañeros. "Con justa razón. Por ejemplo, cuando uno tiene algo, saber si hay remedio para uno defenderse y estar tranquila. Digamos, las que tenemos compañero, es muy importante, porque hay hombres que les gusta picar aquí y allá, y vayamos a ver que enfermedad cogen por allá."

Cuando la mujer cuenta con el apoyo de la pareja, los hijos y los padres, 
(especialmente la madre) va a tener condiciones que facilitan la utilización de los servicios de citología.: "Pues, uno ve que en ese sentido él también me apoyaba, vaya, o si no, vaya y saque plata y vaya y compre su droga".

La percepción de una buena calidad en el servicio fue considerada una motivación para la toma del examen.: “(...) cuando hay una buena atención y cuando, por decir hay comunicaciones de la enfermera y el paciente, pues lógico que uno se va relajando, va teniendo como más confianza, entonces para mi fue una experiencia buena". Las mujeres expresaron encontrar diferencias de calidad de acuerdo al tipo de institución: las mayores de 35 años e incluso las afiliadas al régimen subsidiado prefieren acudir a consulta privada o Profamilia, porque la atención es buena, la explicación del examen es completa y la entrega de resultados es más rápida.

Adecuada información. Las mujeres afiliadas al sistema de salud, recibieron información en la consulta médica, talleres y charlas en los servicios; las mujeres no aseguradas recibieron información a través de los medios de comunicación masiva, el perifoneo, los plegables y las carteleras.

\section{DISCUSIÓN}

En el presente estudio se encontró que el proceso de objetivación frente al examen citológico, se produce de una manera relativamente satisfactoria para la mirada de la salud pública, ya que el concepto evoca en las mujeres ideas que se acoplan, con mayor o menor precisión, con el discurso médico. Las mujeres definen la citología como un medio para detectar infecciones, pre-cáncer y cáncer. La asociación existente entre cáncer del cuello uterino, infecciones vaginales y falta de higiene coincide con lo reportado en otros estudios realizados con mujeres latinas (18). En otros estudios (19), no se encontró que las mujeres latinas de zonas marginales expresaran con suficiente claridad que el cáncer de cuello uterino es una enfermedad prevenible, ni que utilizaran el término pre-cáncer; este término ha sido considerado de difícil comprensión para las mujeres (20).

La práctica de la citología define un mayor o menor riesgo para cáncer en tres categorías básicas: mujeres que nunca se han hecho la citología, aquellas que se la han realizado alguna vez pero hace más de tres años, y las que se la han realizado en los últimos tres años. Para las mujeres que nunca se han realizado la citología en la vida, que todavía ocupan un porcentaje relativamente importante en Colombia (8), los sentimientos de temor y vergüenza han sido una razón primordial no solo en este país (7) como se reafirmó en el presente estudio, sino 
además, en otras comunidades inmigrantes de Estado Unidos (21). En mujeres colombianas, las iniciativas de prevención y autocuidado se expresan en actitudes de sumisión y represión debido al poder ejercido por el cónyuge (22); así lo expresaron algunas de las mujeres de este estudio.

A diferencia de otros estudios (17), en el presente, no se encontró una relación univoca entre el conocimiento y la práctica de la citología. Ante una decisión como la práctica de la citología son muchos los elementos de orden emocional y valorativo que se movilizan de forma independiente de los contenidos adquiridos en un plano puramente racional. Desde la teoría de las representaciones sociales, este hecho podría definirse como un problema en el anclaje de los contenidos. El hecho de conocer la utilidad o las ventajas del examen, a través del proceso de objetivación entra en conflicto con los procesos de culpa que implica el reconocimiento de una vida sexual activa y posible asociación causal con una enfermedad tradicionalmente asociada a la muerte.

Las mujeres del estudio asocian el cáncer con infección; sin embargo no comprenden que ésta pueda haberse generado a partir de una relación sexual antigua. Dentro de la representación del cáncer de cuello uterino de las mujeres predomina una idea de contagio propia de las infecciones agudas, que producen signos y síntomas en un periodo corto de tiempo. En consecuencia, ellas asumen que quienes no han tenido relaciones sexuales por un largo periodo de tiempo no tienen necesidad de realizarse la citología. Desde el punto de vista del anclaje, podría pensarse que existe un proceso de resistencia emocional a la idea de contagio en ausencia de actividad sexual reciente o relativamente reciente. La valoración causal de la relación contagio-sexualidad pareciera atenuarse cuando la mujer ya no tiene vida sexual activa. Este hecho podría también expresarse como un "vacío en la percepción del riesgo" (23) presente en las mujeres mayores de 45 años, quienes probablemente tienen una vida sexual menos activa que las mujeres jóvenes.

Entre las mujeres mexicanas residentes en el Paso, Texas, Estados Unidos se encontró una baja participación al tamizaje entre mujeres mayores de 45 años (24). Por el contrario, en un estudio realizado en el Estado de Morelos, México (17) la prevalencia del antecedente de citología en mujeres entre 45-49 años de edad fue 4,5 veces mayor en comparación con las menores de 20 años. Sin embargo, en este último estudio, el principal motivo para la realización de la citología en este grupo de edad fue la presencia de síntomas ginecológicos urinarios o abdominales, lo cual permite inferir que hay un vacío en la percepción 
del riesgo por cuanto un bajo porcentaje de mujeres consultan en ausencia de síntomas.

Finalmente, en este estudio se encontró que las mujeres afiliadas al régimen subsidiado y las no aseguradas hacen un gran énfasis en la calidad de los servicios de salud como barrera o como factor de motivación para su realización. La tramitología, la falta de calidez en el trato, la falta de oportunidad y la percepción de un cierto caos administrativo se convierten en su conjunto en la barrera sobre la cual hacen más énfasis para el cumplimiento del esquema de tamizaje. Otro estudio realizado en Bogotá propone que aunque las coberturas sean altas, esto solo es importante si el programa cumple el ciclo completo "desde la captación de la mujer hasta la atención adecuada y oportuna cuando se diagnóstica la neoplasia” (25).

Por los hallazgos de este estudio es claro que en el municipio de Soacha hay un problema en la calidad que podría ser común a otros municipios de Colombia. Una limitación básica en este estudio es el hecho de que Soacha tiene unas características particulares por su cercanía con Bogotá que hace que un porcentaje importante de mujeres trabajen y se realicen la citología en el Distrito Capital. En este sentido, es necesario, hacer triangulación de los resultados mediante una encuesta poblacional y posteriormente el desarrollo de una propuesta de reorganización de los servicios para el control en cáncer de cuello uterino en el municipio de Soacha que sirva como modelo de gestión para otros municipios de Colombia

Agradecimientos. A Maria Benilda Melo, enfermera en año social obligatorio en el Instituto Nacional de Cancerología. A las mujeres de Soacha. A Deyanira Rivera Romero, María Elena Alarcón Ovalle y Dalia Cristina Rojas García por el desarrollo de los grupos focales. A la Liga Colombiana contra el Cáncer por su apoyo logístico. Esta investigación fue realizada con recursos de inversión del Instituto Nacional de Cancerología, de la Universidad Externado de Colombia y de Colciencias. Igualmente se contó con el apoyo de la Empresa de Salud de Soacha, la Secretaría de salud de Soacha y con la participación de la Gobernación de Cundinamarca.

\section{REFERENCIAS}

1. Ferlay J, Bray F, Pisani P, Parkin DM. GLOBOCAN 2002 Cancer Incidence, Mortality and Prevalence Worldwide IARC Cancer Base No. 5, version 2.0 IARC Press, Lyon; 2004. 
2. Murillo RH. La prevención del cáncer más allá de las pruebas de tamizaje. Revista Colombiana de Cancerología. Junio 2003; 7 (2): 23-33

3. Programa par una Tecnología Apropiada en Salud (PATH). Organización Panamericana de la Salud (OPS). Planificación de programas apropiados para la prevención del cáncer cérvico uterino. Washington, D.C.: OPS; 2002.

4. Sankaranarayanan R, Budukh MA, Raijkumar R. Effective screening programs for cervial cancer in low and middle-income developing countries. Bull WHO. 2001; 79(10):954-961.

5. Lazcano PE, Castro R, Allen R, Najera P, De Ruiz PA, Hernández AM. Resistencias a la utilización de programas de detección oportuna de cáncer cérvico-uterino. En: Cáncer cérvico-uterino: diagnóstico, prevención y control. Lazcano-Ponce E, Alonso P, Hernández M México: Editorial Médica Panamericana; 2000.

6. Geisinger K. Obstacles to reducing cervical cancer in Vietnam. Am J Clin Pathol 2004; 121:313-314.

7. Ministerio de Salud, Centro Nacional de Consultoría. Conocimientos, actitudes y prácticas en detección temprana de cáncer cérvico-uterino y de mama. II Estudio Nacional de Factores de Riesgo de Enfermedades Crónicas (ENFREC II). Tomo IV. Bogotá D. C.: Ministerio de Salud; 1999.

8. Ojeda G, Ordóñez M, Ochoa L, Samper B, Sánchez F. Salud sexual y reproductiva: Resultados Encuesta Nacional de Demografía y Salud 2005. Asociación Probienestar de la Familia Colombiana (Profamilia).. Patrocinado por Profamilia, Bienestar Familiar, United State Agency for Internacional Development, Ministerio de la Protección Social; Noviembre de 2005.

9. Realpe C, Escobar GM, Largo B, Duque B. Utilización del programa de promoción y prevención en las empresas promotoras de salud y administradoras del régimen subsidiado de Manizales, Colombia, 2001-2002. Colombia Médica 2002; 33 (3): 102-107.

10. Ramírez H, Vélez S, Vargas V, Sánchez C, Lopera J. Evaluación de la cobertura en aseguramiento y accesibilidad a los servicios de salud de los afiliados al régimen subsidiado en el municipio de Medellín. Colombia Médica 2001; 32: 10-13.

21. Martínez P, Rodríguez L, Agudelo C. Equidad en la política de la reforma del sistema de salud. Rev. Salud Pública (Colombia). 2001; 3: 19-39.

12. Viveros M. La noción de representación social y su utilización en los estudios sobre salud y enfermedad. Rev Colombiana de Antropología. 1993; 23:238-260.

13. Jodelet D. La Representación Social: Fenómenos, concepto y teoría. En: Moscovici S. Psicología Social. Barcelona: Paidós; 1986.

14. Mora M. La teoría de las representaciones sociales en Sergei Moscovici. Athenea Digital 2002; 2:1-25. 
196 REVISTA DE SALUD PÚBLICA · Volumen 8 (3), Noviembre 2006

15. Strauss A, Corbin J. Bases de la Investigación Cualitativa. Técnicas y Procedimientos para desarrollar la teoría fundamentada. Medellín: Ed. Universidad de Antioquia; 2002. pp. 219-235.

16. Taylor S, Bogdan R. Introducción a los métodos cualitativos de investigación. Barcelona: Paidós Ibérica; 1992. p.343.

17. Lazcano-Ponce E. Factores que determina la participación de cáncer cervical en el estado de Morelos. Salud Pública de México 1999; 41:278-285.

18. Chávez LR, Hubbell A, Mc Mullin JM, Martinez RG, Mishra SI. Structure and meaning in models of breast and cervical cancer risks factors: a comparison of perceptions among Latinas, Anglo Women, and Physicians. Medical Anthropology Quarterly 1995; New Series 9(1):40-74.

19. Bingham A, Bishop A, Coffey P, Winkler J, Bradley J, Dzuba I, et al. Factors affecting utilization of cervical cancer prevention services in low- resource settings. Salud Pública de México 2003; 43 Supp3: 408-16.

20. IARC. International Agency for Cancer Research. Cervix cancer Screening Meeting. Lyon: IARC; 2004.

21. Hubbell FA, Chavez LR, Mishra SI, Valdez RB. Beliefs about Sexual Behavior and Other Predictors of Papanicolau Smear Screening Among Latinas and Anglo Women. Arch Intern Med 1996; 156:2353-2358.

22. Presidencia de la República de Colombia, Instituto Nacional de Cancerología. Pase a la vida. Guía para el diseño de una estrategia local de comunicación del programa de detección precoz y control del cáncer de cuello uterino. Bogotá: Instituto Nacional de Cancerología; 1995.

23. Douglas M. La aceptabilidad del riesgo según las Ciencias Sociales. Buenos Aires: Paidós; 1996.

24. Suarez L, Lloyd L, Weiss N, Rainbolt T, Pulley L. Effect of Social Networks on Cancer. Screening Behavior of Older Mexican-American Women. J Nat Cancer Institute. 1994; 86 (18):775-779.

25. Lucumi DI, Gómez LF. Accesibilidad a los servicios de salud de citología reciente de cuello uterino en una zona urbana de Colombia. Rev Esp Salud Pública. 2004; 78: 367-377. 